\title{
Modenselektive Eigenschaften einer planparallelen dielektrischen Platte als Reflektor eines Laserresonators*
}

\author{
G. Gehrer und D. Röss \\ Mitteilung aus dem Zentrallaboratorium der Siemens \& Halske AG., München \\ (Z. Naturforschg. 20 a, 701--705 [1965]; eingegangen am 24. Februar 1965)
}

\begin{abstract}
Die Resonanzeigenschaft eines dielektrischen Etalons führt bei seiner Anwendung als Reflektor eines Laserresonators zu einer starken axialen und transversalen Modenselektion. Es treten näherungsweise nur solche Moden auf, die Moden des Etalons selbst sind. Die Temperaturabhängigkeit der Verstärkungslinie führt bei einem Rubinlaser dazu, daß in einem Pumpimpuls mehrere Moden nacheinander emissionsfähig werden. Mit einer planparallelen Glasplatte als Reflektor ist es möglich, in einem Rubinlaser bei Zimmertemperatur nur jeweils einen axialen Mode gleichzeitig anzuregen.
\end{abstract}

\section{Modenselektive, gekoppelte Resonatoren}

In Laserresonatoren schwingen bei hoher Pumpenergie eine große Zahl von Moden. Der Grund hierfür liegt einmal in der großen Linienbreite der benützten Übergänge und in der räumlich großen Ausdehnung der Resonatoren. Andererseits verhindern spektral selektive Prozesse eine Kondensation der Emission in den Mode höchster Güte und Verstärkung. Durch Abkühlen des Lasermaterials kann bei Rubin die Linienbreite verringert werden, und durch Verkleinern der Resonatorlänge wächst der Abstand der axialen Moden. Beide Maßnahmen setzen in einem gewissen Maß die Zahl der schwingenden Moden herab, wobei die Verkürzung des Resonators allerdings auch zu geringeren Laserleistungen führt.

Schon früh hat man daneben nach modenselektierenden Anordnungen unter Verwendung gekoppelter Resonatoren gesucht. Kleinmann und Kisliuk ${ }^{1}$ betrachteten theoretisch einen Resonator mit 4 ebenen und parallelen Spiegeln. Ein entsprechendes Experiment mit sphärischen Spiegeln wurde für den Gaslaser von Skinner und Geusic ${ }^{2}$ durchgeführt. ColLINS und $\mathrm{WHITE}^{3}$ brachten in den Resonator eines Rubinlasers zwei leicht geneigte und gegeneinander um $90^{\circ}$ verdrehte Glasplatten. Sie erzielten hierdurch eine Selektion axialer Moden und eine Verringerung des Strahlungswinkels. Zu ähnlichen Er-

* Auszugsweise vorgetragen auf der Tagung der Deutschen Physikalischen Gesellschaft 1964 in Düsseldorf.

1 D. A. Kleinmann u. P. D. Kisliuk, Bell System Techn. J. 41, 453 [1962].

2 J. G. Skinner u. J. E. Geusic, Opt. Soc. Amer. Meeting 6. Okt. 1962, Rochester N. Y. gebnissen kamen Rothe und MANGER ${ }^{4}$ mit einem Etalon im Resonator, die Neodym in Glas als Lasersubstanz benutzen. Wir haben eine besonders einfache Anordnung beschrieben ${ }^{5}$, bei der ein Spiegel eines gewöhnlichen FABry-Perot-Resonators durch eine planparallele dielektrische Platte ersetzt wird. Diese Anordnung wurde auch von Snitzer ${ }^{6}$ verwendet.

\section{Versuchsanordnung}

Wir haben die Modenselektion dieses Systems mit einem Fabry-Perot-Interferometer untersucht. Abb. 1 zeigt den Aufbau der experimentellen Anordnung.

Ein mit Saphir ummantelter Rubin befindet sich zwischen einem dielektrischen Spiegel und der dielektrischen, planparallelen Platte. Das Reflexionsvermögen des dielektrischen Spiegels ist im Bereich der Rubinlinie wellenlängenunabhängig gleich $98,5 \%$. Die dielektrische Platte besteht aus Glas oder Rutil, je nach dem

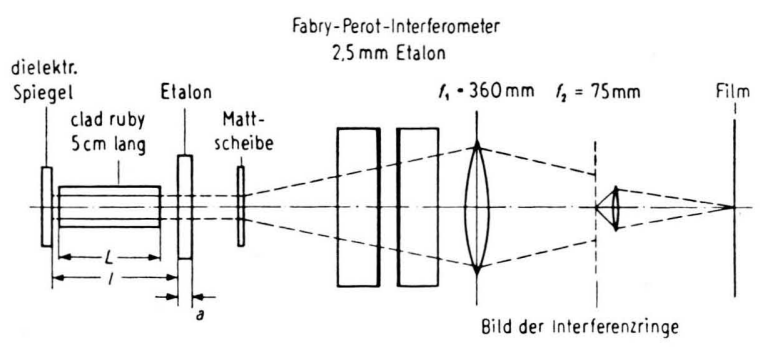

Abb. 1. Versuchsaufbau.

3 S. A. Collins u. G. R. White, Appl. Opt. 2, 448 [1962].

4 H. Manger u. H. Rothe, Phys. Letters 7, 330 [1963].

5 D. Röss, Proc. IEEE 52, 196 [1964].

6 E. Snitzer, Proc. Conf. Quantum Electronics Paris 1963, Columbia University Press, New York 1964, S. 999. 
Grad der angestrebten Reflexion. Für die praktische Anwendung ist eine Glasplatte besonders vorteilhaft, die beidseitig mit einer dünnen Rutilschicht $\left(\mathrm{TiO}_{2}\right)$ bedampft wird. Man verbindet dabei die höhere Reflexion mit der besseren optischen Qualität der Glasplatte. Mehrschichtige Verspiegelungen sind weniger günstig, da ihre Reflexionsmaxima zu breit werden. Bei einem Teil der Versuche wurde vor dem FABRy-PerotInterferometer der Laserstrahl aufgestreut, um das ganze Interferometer auszuleuchten. Jede im Laserstrahl enthaltene Frequenz erzeugt in der Brennebene der Linsenanordnung in bekannter Weise ein konzentrisches Ringsystem.

\section{Modenselektion einer dielektrischen Platte}

Die Transmission $T$ und das Reflexionsvermögen $R$ der benützten dielektrischen Platten sind für ebene, senkrecht auffallende Wellen periodische Funktionen der Frequenz der auffallenden Welle. Diese Abhängigkeit erklärt sich durch Interferenz der an der Vorder- und Rückseite der Platte reflektierten Wellen. Es gilt in bekannter Weise:

$$
\begin{gathered}
T=\frac{1}{1+\left[4 r /(1-r)^{2}\right] \sin ^{2}(2 \pi n a / \lambda)}, \\
R=1-T .
\end{gathered}
$$

Hierbei ist $r$ der Energiereflexionsfaktor des Plattenmaterials :

$$
r=[(n-1) /(n+1)]^{2} .
$$

$n$ ist der Brechungsindex der Platte, $a$ die Plattendicke und $\lambda$ die Wellenlänge. Aus (1) und (2) folgt, $\mathrm{da} \beta$

$$
R_{\min }=0 \quad \text { und } \quad R_{\max }=4 r /(1+r)^{2} .
$$

Demnach können senkrecht auffallende Wellen ganz bestimmter Frequenz die Platte ohne Reflexion passieren. Der Frequenzabstand dieser äquidistanten Reflexionsminima (und auch der Reflexionsmaxima) beträgt nach (1)

$$
\Delta v=c / 2 n a .
$$

Das maximale Reflexionsvermögen im Falle einer Glasplatte (BK 7, $n=1,51$ ) beträgt nach Gl. (4) etwa $15 \%$, im Falle einer Rutilplatte $(n=2,7)$ etwa $60 \%$. Für eine Glasplatte von 2,6 mm Dicke liegt der Abstand der Reflexionsminima nach Gl. (5) bei etwa $0,62 \AA$. Dieser Wert ist mit der Halbwertsbreite der Rubinlinie von $2-3 \AA$ bei Zimmertemperatur zu vergleichen. In Abb. 2 ist unter der Rubinfluoreszenzlinie der Verlauf des Reflexionsvermögens für die Glasplatte von 2,6 mm Dicke aufgezeichnet.
Ersetzt man in einem gewöhnlichen FABRY-PERotLaserresonator einen Spiegel durch eine solche planparallele Glasplatte, so haben die einzelnen axialen Moden durch die Wellenlängenabhängigkeit des Reflexionsvermögens unterschiedliche Verluste. Moden hoher Reflexion werden dadurch selektiert. In

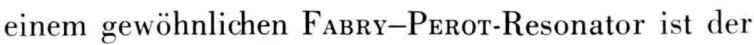
Frequenzabstand der axialen Moden durch

$$
\Delta v=c / 2 n l
$$

gegeben, wobei $n l$ die optische Resonatorlänge ist. So beträgt beispielsweise für eine optische Resonatorlänge von $8,5 \mathrm{~cm}$ der Modenabstand $0,03 \AA$. Bei Benützung der Glasplatte hängt die Phase der reflektierten Welle wie deren Amplitude von der Wellenlänge der einfallenden Welle ab. Die axialen Moden sind nicht mehr äquidistant. KLeinmann und Kisliuk haben aber gezeigt, daß Gl. (6) für die in der Emission auftretenden Moden näherungsweise richtig ist, wenn $n l$ den optischen Weg vom dielektrischen Spiegel zu der dem Rubin zugekehrten Oberfläche der Glasplatte bedeutet.

Bei der Verwendung einer modenselektiven Platte als Reflektor bestimmen $g(v)$ und $R(v)$ die Schwellenergie der einzelnen axialen Moden. Die Schwellbedingungen für das Auftreten eines axialen Modes lautet:

$$
e^{2 \alpha L} R \approx 1 .
$$

Hierbei ist $\alpha$ der Verstärkungsfaktor, $L$ die Länge des Lasermaterials und $R$ der Energiereflexionsfaktor der Glasplatte. Der Reflexionsfaktor des zweiten Spiegels ist zu 1 angenommen. Für $\alpha$ gilt ${ }^{7}$

$$
\alpha=\left(n_{2}-n_{1}\right) \frac{h v c^{2}}{8 \pi h v^{3} t_{\mathrm{sp}}} g(v),
$$

wobei $n_{2}-n_{1}$ die Inversion, $t_{\mathrm{sp}}$ die spontane Lebensdauer der angeregten Rubinatome und $g(v)$ die Form der Rubinfluoreszenzlinie ist. Hiermit berechnet sich nach Gl. (7) die zum Einsetzen einer Schwingung nötige Inversion zu

$$
n_{2}-n_{1}=\frac{1}{2 L} \frac{8 \pi h v^{3} t_{\mathrm{sp}}}{h v c^{2}} \frac{1}{g(v) / \ln [1 / R(v)]} .
$$

An die Stelle der Rubinfluoreszenzlinie $g(v)$ im Falle

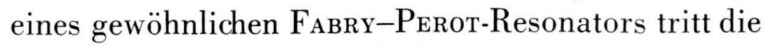
Funktion $g(v) / \ln [1 / R(v)]$, die als scheinbare Linienform bezeichnet werden kann. Diese scheinbare Linie müßte der Rubin in einem gewöhnlichen FABRYPеrot-Resonator haben, um die gleiche Emission wie

\footnotetext{
7 A. Yariv u. J. P. Gordon, Proc. IEEE 51, 4 [1963].
} 
G. Gehrer und D. Röss, Modenselektive Eigenschaften einer planparallelen dielektrischen Platte als Reflektor eines Laserresonators (S. 701).

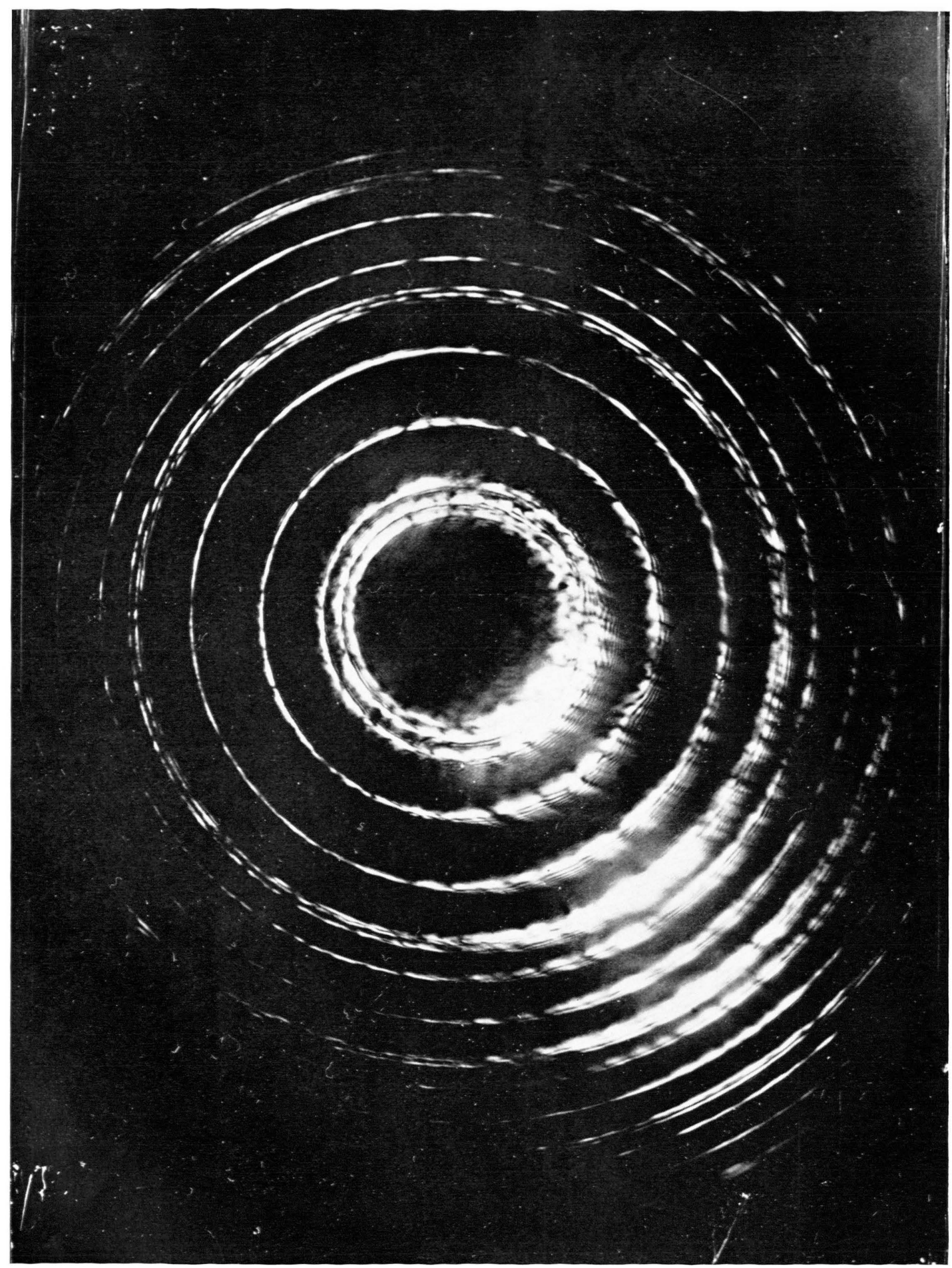

Abb. 5. Spektrum der Laserstrahlung bei hoher Auflösung Abstand der Interferometerplatten $20 \mathrm{~mm}$. Als Resonatorspiegel wurde eine 2,6 $\mathrm{mm}$ dicke Glasplatte benutzt. 

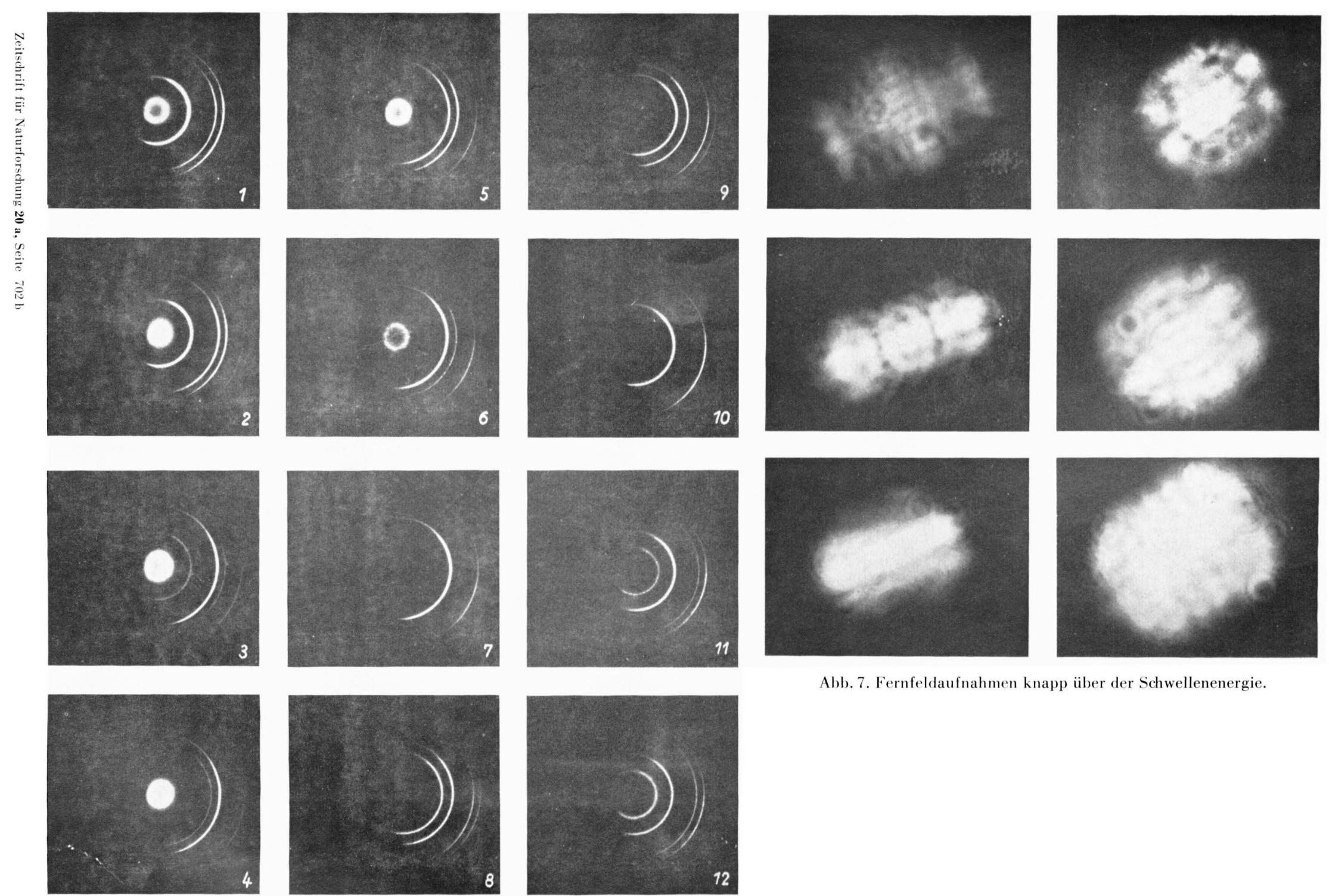

Abb. 7. Fernfeldaufnahmen knapp über der Schwellenenergie.

Abb. 4. Emissionsspektrum eines Rubinlasers mit Etalon-Reflektor. FaвrY-Pевот-Ringe bei Verwendung einer Glasplatte von 5,4 mm Dicke; Abstand der Interferometerplatten 2,5 mm. Die Rubintemperatur steigt von Aufnahme zu Aufnahme um $2{ }^{\circ} \mathrm{C}$. 
in dem untersuchten Resonator zu ergeben. In Abb. 2 ist unter dem Reflexionsvermögen diese Frequenzabhängigkeit aufgetragen, darunter die Dichte der axialen Moden in einem gewöhnlichen $\mathrm{F}_{\text {ABRY-PERoT- }}$ Resonator von $8,5 \mathrm{~cm}$ optischer Länge. Moden, für die die scheinbare Linie ein Maximum hat, be-

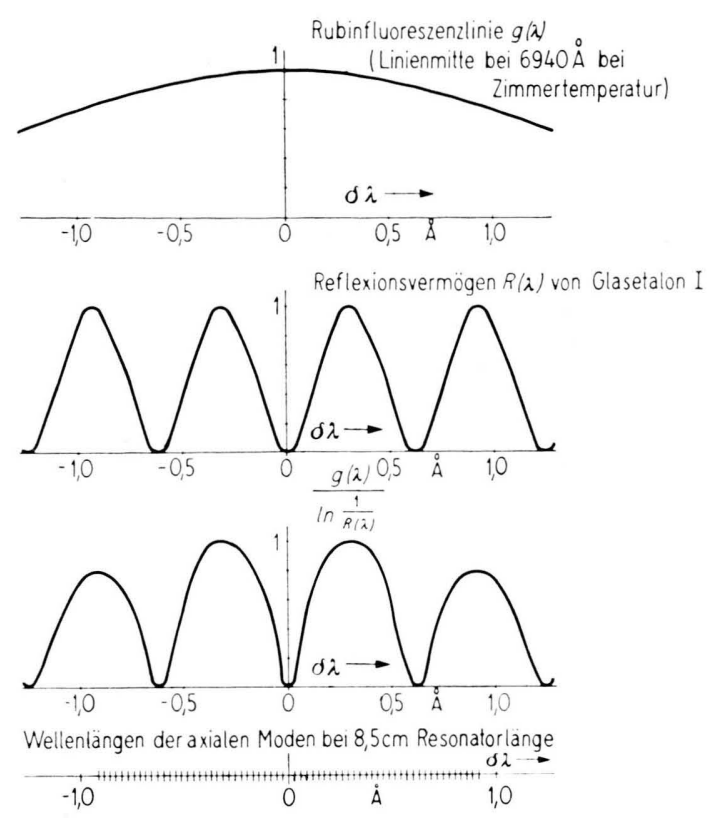

Abb. 2. Rubinlinie, Reflexionsvermögen, scheinbare Linienform und Spektrum der axialen Moden. Die Mitte der Rubinlinie fällt mit einem Reflexionsminimum zusammen.

nötigen die geringste Inversion zum Einsatz von Schwingungen. Moden in der Linienmitte treten in dem Beispiel trotz großer Verstärkung nicht auf, da hierfür die Spiegelrückkopplung fehlt. Die Emission setzt in 2 Bändern am Ort der beiden höchsten Maxima ein. Die Frequenzen der Maxima sind wegen $g(v)$ nicht genau äquidistant; sie entsprechen etwa den Moden des Etalons selbst. Wir haben im Experiment bei einer Serie von Aufnahmen die Temperatur des Rubinkristalls schrittweise erhöht. Hierbei wandert die Rubinlinie über eine ganze Reihe von Reflexionsmaxima und Minima hinweg. Während in Abb. 2 angenommen ist, daß ein Reflexionsminimum mit der Rubinlinienmitte zusammenfällt, wird dies in Abb. 3 für ein Reflexionsmaximum angenommen. Hier setzt die Emission in einem einzigen Band in der Linienmitte oder bei hoher Pumpenergie in 3 äquidistanten Bändern ein.
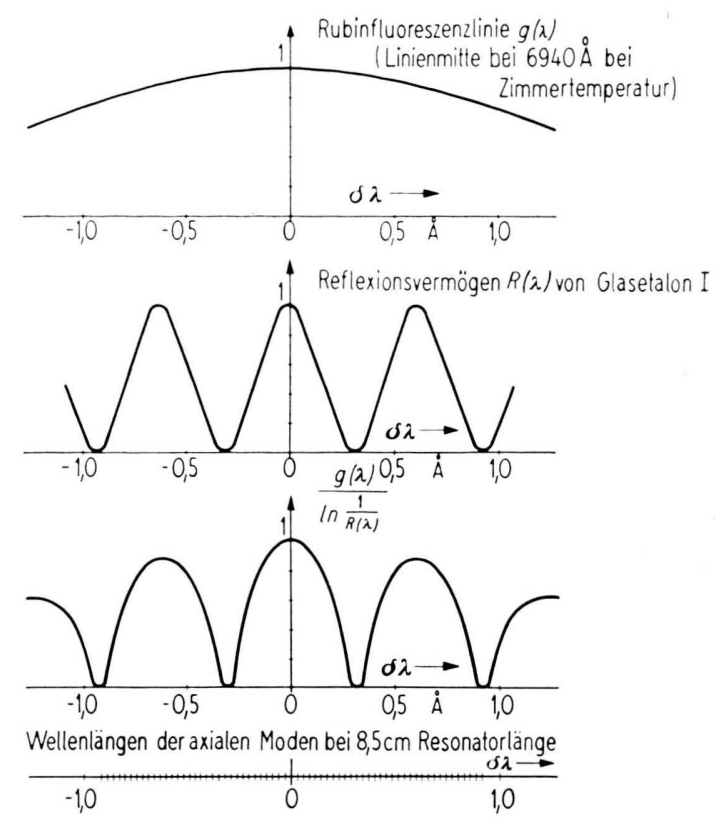

Abb. 3. Rubinlinie, Reflexionsvermögen, scheinbare Linienform und Spektrum der axialen Moden. Die Linienmitte fällt mit einem Reflexionsmaximum zusammen.

\section{Experimentelle Ergebnisse}

Zur Untersuchung der jeweiligen Emission wurde für das Interferometer ein Plattenabstand von $2,5 \mathrm{~mm}$ gewählt. Dabei beträgt das Dispersionsgebiet etwa $1 \AA$. Die Auflösung reicht nicht aus, um einzelne axiale Moden zu trennen. Wie aus Abb. $4{ }^{*}$ zu sehen ist, werden die Ringsysteme von Emissionsbändern, die den einzelnen Reflexionsmaxima entsprechen, beobachtet. Die Temperatur nimmt von Aufnahme zu Aufnahme um etwa $2{ }^{\circ} \mathrm{C}$ zu. Dabei verschiebt sich die Rubinlinienmitte um etwa 0,15 ̊. Die Pumpenergie beträgt $200 \mathrm{Ws}$.

In den ersten 3 Aufnahmen von Abb. 4 treten 4 Ringe auf, deren Radien von Bild zu Bild gleich sind. Zu sehen sind jeweils 2 Ringsysteme. Zu Ringsystem 1 soll der zweite und vierte Ring gehören, zu Ringsystem Nr. 2 der erste und dritte Ring. Bei der dritten Aufnahme ist das erste Ringsystem bereits intensitätsschwach, da sich hier die Rubinlinie über das zugehörige Reflexionsmaximum hinweggeschoben hat. In der vierten Aufnahme ist das zweite Ringsystem noch mit gleicher Intensität wie vorher vorhanden, aber das erste Ringsystem ist verschwunden, und ein neues drittes Ringsystem ist aufgetaucht. In

* Abb. 4, 5 und 7 auf Tafel S. 702 a, b. 
der fünften Aufnahme haben das zweite und dritte Ringsystem gleiche Intensität. Ebenso können die weiteren Aufnahmen erklärt werden. Ein Ringsystem auf der letzten Aufnahme entspricht der Emission an der Stelle des fünften Reflexionsmaximums.

Abb. 5 zeigt eine Aufnahme mit besonders hoher Auflösung. Der Abstand der dielektrisch verspiegelten Interferometerplatten beträgt $20 \mathrm{~mm}$. Linien im Abstand von $100 \mathrm{MHz}$ werden noch getrennt. Die Frequenzen in der Emission überstreichen einen Bereich größer als das Dispersionsgebiet von $0,12 \AA$. Der Abstand der Interferometerplatten wurde so gewählt, daß die auftretenden Ringsysteme nicht aufeinanderfallen. Ohne modenselektiven Reflektor wäre eine Aufnahme mit so hoher Auflösung nicht möglich. Die optische Resonatorlänge beträgt $16 \mathrm{~cm}$. Damit liegen 8 axiale Moden im Dispersionsgebiet. Die Pumpenergie ist $250 \mathrm{Ws}$. Es treten 3 Ringsysteme auf, die wie vorher zu 3 Reflexionsmaxima gehören. Das Ringsystem, das dem mittleren Reflexionsmaximum entspricht, enthält die Frequenzen von drei Moden. Da bei dieser Aufnahme keine Mattscheibe vor dem Interferometer benützt wurde, überlagert sich dem Ringsystem noch eine Fernfeldstruktur. Man erkennt den Einfluß der Materialinhomogenität; die Frequenzen sind etwas abhängig von der Lage im Fernfeld. Auch hier beobachtet man bei Temperaturänderung des Rubinkristalls das Verschwinden bzw. Auftreten neuer Emissionsbänder. Mit wachsender Pumpenergie werden diese Emissionsbänder breiter; die FABRY-PеRот-Ringe überlappen sich schließlich. Bei konstanter Pumpenergie und wachsender Resonatorlänge nimmt die Zahl der Moden in den einzelnen Emissionsbändern rasch zu, so daß die Auflösung zu ihrer Trennung nicht mehr ausreicht.

\section{Zeitliches Emissionsverhalten}

Bei geringer Plattendicke $(2,6 \mathrm{~mm})$ beobachtet man bei guter Justierung nur Moden in einem Reflexionsmaximum, solange die Pumpenergie unterhalb der doppelten Schwellenergie bleibt. Dabei ist jeweils nur ein axialer Mode zu einem bestimmten Zeitpunkt emissionsfähig. Aufnahmen des zeitlichen Verlaufs sind in Abb. 6 dargestellt (siehe auch ${ }^{5}$ ). Man beobachtet nahe der Schwelle regelmäßige Impulsfolgen oder stark gedämpfte Relaxationsschwingungen in jeweils einem einzigen axialen Mode. Auf manchen Aufnahmen ist eine Modulation im $\mathrm{MHz}$ Bereich erkennbar, die auf Schwebungen zwischen

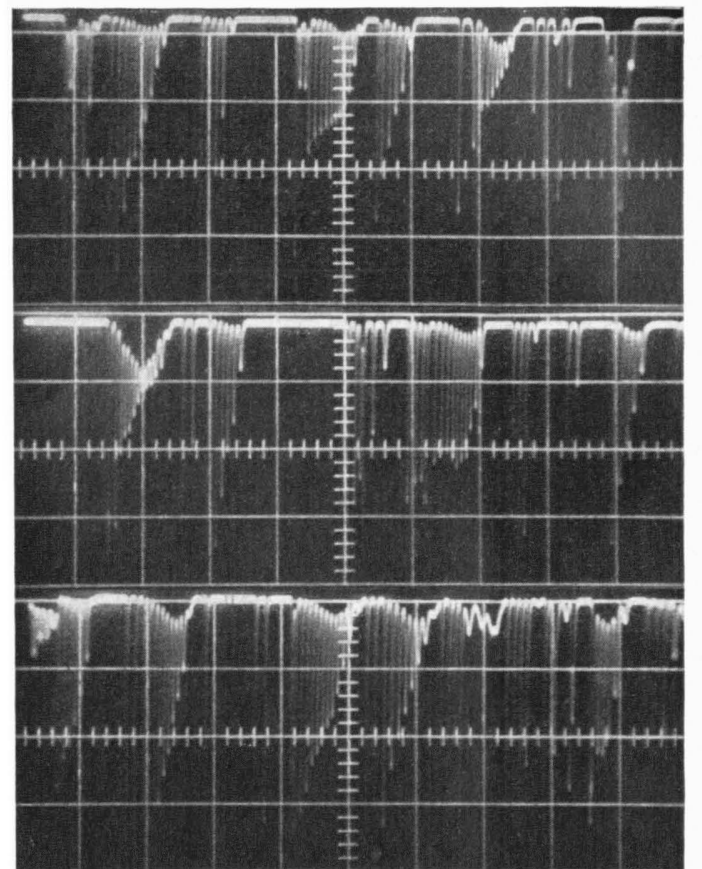

Abb. 6. Zeitlicher Verlauf der Emission (negative Ordinatenrichtung) 2,6 mm dicke Glasplatte; $100 \mu \mathrm{s} /$ Einheit.

transversalen Moden geringer Zahl zurückzuführen ist. Die Emission eines Mode reißt nach etwa $100 \mu \mathrm{s}$ ab. Bei höherer Pumpenergie beginnt sie, zeitlich deutlich abgesetzt, im nächsten axialen Mode. Man erhält schließlich eine ganze Reihe isolierter Ausbrüche der Emission, die unregelmäßiger werden und schließlich überlappen. In diesen Aufnahmen wandert die Rubinlinie während eines Pumpimpulses über die Kuppe eines Reflexionsmaximums. Durch die thermische Drift der Rubinlinie werden die axialen Moden in der Nähe des Reflexionsmaximums zeitlich hintereinander so bevorzugt, daß sie getrennt nacheinander schwingen. Die Zeitpunkte, in denen sich die Linienmitte zwischen zwei axialen Moden befindet, sind für die Emission relativ ungünstig, so daß sie aussetzt. Dieser Effekt ist die Folge der größeren Krümmung der scheinbaren Linienform im Vergleich zur tatsächlichen Rubinlinie. Man beobachtet aber auch in einfachen Rubinlasern eine periodische Amplitudenmodulation der Spikes bei niedriger Modendichte ${ }^{8}$, besonders wenn, wie in unseren hier verwendeten ellipsoidalen Pumpanordnungen, störende Unsymmetrien vermieden werden.

${ }^{8}$ D. Röss, Appl. Opt. 3, 259 [1964]. 
Abb. 7 zeigt einige Fernfeldaufnahmen des Laserstrahls. Die ausgeprägte Modenstruktur zeigt, daß der untersuchte Resonator auch die transversalen Moden stark selektiert. Der Öffnungswinkel des Laserstrahls ist klein und erreicht den durch die Beugungsgrenze für den angeregten Modentyp gegebenen Wert. Wegen der hohen Streuverluste von Rubin sind die Maxima der Modenbilder diffus.

\section{Folgerungen}

Mit dem hier untersuchten Resonator ist es möglich, die spektrale Emission in einfacher Weise auf einen kleineren Bereich zu konzentrieren und damit eine höhere Intensität pro Wellenlängenintervall und Raumwinkel zu erzielen als in einfachen Resonatoren. Die erzielte regelmäßige Emission wirft weiteres Licht auf den Mechanismus der Laseremission und kann helfen, die unregelmäßige Emission in gewöhnlichen
FabRY-Perot-Resonatoren zu verstehen; sie wird zu einem Teil durch das thermische Herauswandern der schwingenden Moden aus dem schwingungsfähigen Frequenzbereich erzeugt. Es zeigt sich, daß es grundsätzlich nicht möglich ist, einen Rubinlaser im Impulsbetrieb wesentlich länger als einige $100 \mu \mathrm{s}$ in einem Mode schwingen zu lassen. Denkbar ist die Verwendung mehrerer dielektrischer Platten als Ersatz für den hochreflektierenden Spiegel oder eine Kombination von 2 aufeinandergelegten Glasplatten von verschiedenem Brechungsindex als Reflektor, um die Emission weiter einzuengen und zusätzliche Emissionsbänder zu unterdrücken.

Eine praktisch wichtige Eigenschaft dielektrischer Platten ist ihre Resistenz gegen Laserstrahlung hoher Leistung.

Für seine Hilfe bei den experimentellen Arbeiten danken wir Herrn W IEBERGER.

\title{
Zur Entstehung von Nadelkristallen beim Aufdampfen
}

\author{
Wolf-Udo Wagner \\ Physik-Department der Technischen Hochschule München \\ (Z. Naturforschg. 20 a, 705-712 [1965] ; eingegangen am 24. Dezember 1964)
}

\begin{abstract}
Calculations are presented of the kinetics of beginning whisker formation from the gaseous phase. This is done for a simple mechanism involving the interaction of spiral growth about a dis. location and impurities. The radius of a whisker is found to be in most cases inversely proportional to the concentration of impurities in the vapour. Formulae are given for the transition from a simple growth spiral to the whisker. The particle density on the surface is computed. Reasons for the assumption of a quasistationary treatment are given. The expressions are modified to include the case of finite dwell periods of the particles.
\end{abstract}

Von einer ganzen Reihe von Substanzen können durch Aufdampfen Whiskers erzeugt werden ${ }^{1}$. Soweit die Nadelkristallbildung nicht mit der Anisotropie der jeweiligen Kristallstruktur einfach gedeutet werden konnte, wurde anderweitig nach Mechanismen gesucht, die das bevorzugte Wachstum in nur einer Richtung erklären sollen. Zur Deutung der Entstehung von KCl-Whiskers aus der Dampfphase wurde unlängst eine relativ einfache Vorstellung entwickelt ${ }^{2}$, welche die Nadelkristallbildung in Anlehnung an einen Vorschlag von Amelinckx ${ }^{3}$ aus dem Zusammenwirken von Wachstumsspiralen mit Spuren von

1 Siehe die Zusammenfassung bei F. R. N. Nabarro u. P. J. JACkson, in Growth and Perfection of Crystals, John Wiley \& Sons, Inc., New York 1958, p. 44 ff. (organischen) Verunreinigungen erklärt. In dieser Arbeit wird nun die Kinetik dieses Prozesses behandelt. Die Ergebnisse werden auf die Beobachtungen bei KCl-Whiskers ${ }^{2}$ angewendet.

Als Ausgangssituation sei eine reine Einkristalloberfläche gegeben, z. B. eine (100)-Ebene bei $\mathrm{KCl}$, die von einer Anzahl solcher Versetzungen mit (teilweisem) Schraubencharakter durchstoßen wird, die zur Ausbildung von Wachstumsspiralen geeignet sind. Zur Zeit $t=0$ wird mit dem Aufdampfen von Gitterbausteinen begonnen. Die Aufdampfgeschwindigkeit $v\left[\mathrm{~cm}^{-2} \mathrm{sec}^{-1}\right]$ sei konstant, dem Dampf sei

2 W.-U. W

3 S. Amelinckx, in Growth and Perfection of Crystals, John Wiley \& Sons, Inc., New York 1958, p. 139 ff. 